\title{
The hypoxic burden of sleep apnoea predicts cardiovascular disease-related mortality: the Osteoporotic Fractures in Men Study and the Sleep Heart Health Study
}

\author{
Ali Azarbarzin ${ }^{1}$, Scott A. Sands ${ }^{1}$, Katie L. Stone ${ }^{2,3}$, Luigi Taranto-Montemurro ${ }^{1}$, \\ Ludovico Messineo ${ }^{1}$, Philip I. Terrill ${ }^{4}$, Sonia Ancoli-Israel ${ }^{5,6}$, Kristine Ensrud ${ }^{7}$, \\ Shaun Purcell ${ }^{1,8}$, David P. White ${ }^{1}$, Susan Redline ${ }^{1}$, and Andrew Wellman ${ }^{1}$
}

\begin{abstract}
${ }^{1}$ Division of Sleep and Circadian Disorders, Brigham and Women's Hospital and Harvard Medical School, Sleep Disordered Breathing Lab, 221 Longwood Avenue, Boston, MA 02115, USA; ${ }^{2}$ Research Institute, California Pacific Medical Center, 550 16th Street, 2nd Floor, San Francisco, CA 94158, USA; ${ }^{3}$ Department of Epidemiology and Biostatistics, University of California, San Francisco, 550 16th St, San Francisco, CA 94158, USA; ${ }^{4}$ School of Information Technology and Electrical Engineering, The University of Queensland, Brisbane, Australia; ${ }^{5}$ Department of Psychiatry, University of California San Diego, 9500 Gilman Drive La Jolla, CA 92093, USA; ${ }^{6}$ Department of Medicine, University of California San Diego, 9500 Gilman Drive La Jolla, CA 92093, USA; ${ }^{7}$ University of Minnesota and Minneapolis Veterans Affairs Health Care System, 1 Veterans Dr, Minneapolis, MN 55417, USA; and ${ }^{8}$ Stanley Center for Psychiatric Research, Broad Institute of MIT \& Harvard, 415 Main St, Cambridge, MA 02142, USA
\end{abstract}

Received 7 May 2018; revised 8 August 2018; editorial decision 17 September 2018; accepted 18 September 2018; online publish-ahead-of-print 30 October 2018

Aims Apnoea-hypopnoea index (AHI), the universal clinical metric of sleep apnoea severity, poorly predicts the adverse outcomes of sleep apnoea, potentially because the AHI, a frequency measure, does not adequately capture disease burden. Therefore, we sought to evaluate whether quantifying the severity of sleep apnoea by the 'hypoxic burden' would predict mortality among adults aged 40 and older.

Methods and results

Conclusion

Keywords
The samples were derived from two cohort studies: The Outcomes of Sleep Disorders in Older Men (MrOS), which included 2743 men, age $76.3 \pm 5.5$ years; and the Sleep Heart Health Study (SHHS), which included 5111 middle-aged and older adults (52.8\% women), age: $63.7 \pm 10.9$ years. The outcomes were all-cause and Cardiovascular disease (CVD)-related mortality. The hypoxic burden was determined by measuring the respiratory event-associated area under the desaturation curve from pre-event baseline. Cox models were used to calculate the adjusted hazard ratios for hypoxic burden. Unlike the AHI, the hypoxic burden strongly predicted CVD mortality and all-cause mortality (only in MrOS). Individuals in the MrOS study with hypoxic burden in the highest two quintiles had hazard ratios of 1.81 [95\% confidence interval $(\mathrm{Cl}) 1.25-2.62$ ] and 2.73 (95\% Cl 1.71-4.36), respectively. Similarly, the group in the SHHS with hypoxic burden in the highest quintile had a hazard ratio of $1.96(95 \% \mathrm{Cl} 1.11-3.43)$.

The 'hypoxic burden', an easily derived signal from overnight sleep study, predicts CVD mortality across populations. The findings suggest that not only the frequency but the depth and duration of sleep related upper airway obstructions, are important disease characterizing features.

Sleep apnoea • CVD mortality • Hypoxic burden • Polysomnography • Apnoea-hypopnoea index

\section{Introduction}

Obstructive sleep apnoea (OSA) is a common disorder characterized by recurrent upper airway collapse during sleep ${ }^{1}$ that leads to sleep fragmentation ${ }^{2}$ and sympathetic activation. ${ }^{3}$ Untreated OSA has been associated with adverse neurocognitive (daytime sleepiness, reduced attention), ${ }^{4-6}$ Cardiovascular disease (CVD) and metabolic complications (hypertension, diabetes, strokes). ${ }^{4,7-14}$ However, the

* Corresponding author. Tel: +1 617732 5619, Fax: +1 617732 7337, Email: aazarbarzin@bwh.harvard.edu

Published on behalf of the European Society of Cardiology. All rights reserved. @ The Author(s) 2018. For permissions, please email: journals.permissions@oup.com. 
impact of OSA on fatal CVD events remains disputed ${ }^{10,15-17}$ due to some inconsistencies in exposure-outcome associations. Indeed, recent evidence suggests a lack of association between positive airway pressure therapy and secondary CVD prevention. ${ }^{15,18}$

Currently, the field of sleep medicine relies on quantifying the frequency of apnoeas and hypopnoeas observed during sleep (apnoeahypopnoea index, $\mathrm{AHI}$ ) to diagnose OSA and quantify its severity. Based on this metric, OSA was found to modestly predict mortality, but results largely were limited to younger to middle-aged men. ${ }^{9,10,16,19,20}$ One interpretation is that the AHI fails to capture the key aspects of OSA that have a deleterious impact on the CV system. Obstructive sleep apnoea is a disorder in which the repeated airway obstructions reduce ventilation and cause blood gas derangement. Therefore, AHI, which provides a simple count of the number of obstructive episodes per hour of sleep, without regard for the duration and the depth of the ventilatory disturbance or blood gas changes, does not fully characterize physiological disturbances. Several observational studies have demonstrated that measures of nocturnal hypoxaemia, such as the percentage of time during sleep with an oxygen saturation below 90\% (TST90), more strongly predict CVD and allcause mortality than the $\mathrm{AHI}^{21-23}$ However, the TST90 and similar metrics characterize not only the intermittent hypoxaemia occurring secondary to obstructive events but also persistent hypoxaemia, such as from chronic obstructive pulmonary disease (COPD) or obesity hypoventilation, which are unrelated to upper airway obstruction and OSA.

Therefore, we sought to develop a severity measure for OSA that quantifies OSA-related hypoxaemia, which we postulate significantly predicts CVD-related mortality after adjusting for routinely measured polysomnography (PSG) indices. Accordingly, we developed a single measure that encapsulates the frequency, duration, and depth of the respiratory-event contribution to arterial hypoxaemia, specifically the oxygen desaturation 'area under the curve' in association with individual apnoeas and hypopnoeas (i.e. OSA specific 'hypoxic burden', see Figure 1 and Methods section). We hypothesized that our measure of the hypoxic burden would predict CVD mortality and all-cause mortality, and tested this hypothesis in the Osteoporotic Fractures in Men Study (MrOS)-Sleep Study ${ }^{24-27}$ and the Sleep Heart Health Study (SHHS). 26,28,29

\section{Methods}

\section{The Osteoporotic Fractures in Men Study}

The first study was the Osteoporotic Fractures in Men Study (MrOS) Sleep Study (http://mrosdata.sfcc-cpmc.net (4 October 2018)). The parent MrOS study is a community-based, prospective cohort study of 5994 men $\geq 65$ years recruited from six centres across the USA (2000-2002) designed to describe the epidemiology of osteoporosis and fractures in older men. ${ }^{2-27}$ To be eligible, individuals had to be capable of walking without assistance and be without bilateral hip replacements. From 2003 to 2005, 3135 men from the MrOS cohort participated in the ancillary MrOS Sleep Study and underwent comprehensive sleep evaluations including full home PSG as described previously. ${ }^{22}$ Participants who reported nightly use of any treatment, including continuous positive airway pressure (CPAP), bilevel positive airway pressure, mouthpiece, or oxygen therapy were excluded from the MrOS Sleep Study. Of the 3135 participants who completed the sleep study, the PSGs of 2907 met initial study quality criteria and were available on the National Sleep Research Resource website (http://sleepdata.org (4 October 2018)) and analysed. Ethical approval was obtained from local institutional review boards, and all participants provided informed consent.

In-home sleep studies using one night of unattended PSG (Safiro, Compumedics, Inc., Melbourne, Australia) were performed with recording of central electroencephalography, bilateral electrooculography, chin electromyography, electrocardiogram, nasal pressure and thermistor (for airflow measurement), chest and thoracic inductance plethysmography, finger pulse oximetry, body position, and leg movements. ${ }^{30}$ The respiratory events that were used to identify changes in oxygen saturation included all apnoeas and hypopnoeas regardless of desaturation (scored on the basis of amplitude changes only). Apnoeas were identified if thermistry airflow was absent or nearly absent for at least $10 \mathrm{~s}$. Hypopnoeas were identified when there was at least $30 \%$ reduction in airflow (by thermistry or nasal pressure) or thoracoabdominal movement for at least $10 \mathrm{~s}$. Events also were linked to associated desaturation for calculation of the $\mathrm{AHI}$, which was defined as the number of all apnoeas plus hypopnoeas associated with a $\geq 3 \%$ decrease per hour of sleep. $\mathrm{SpO}_{2}$ signals were captured by fingertip pulse oximeters (Nonin, Minneapolis, MN, USA) sampled at $1 \mathrm{~Hz}$.

Covariates and outcomes were based on measurements at the clinic exam, questionnaires and adjudication of reported events. Participants completed questionnaires including information on demographics, medical history, smoking status, and alcohol use. A history of the following medical conditions was gathered: concurrent CVD (including congestive heart failure, coronary heart disease, cerebrovascular disease, peripheral vascular disease, claudication, myocardial infarction, angina, and transient ischaemic attack), stroke, diabetes mellitus, renal failure, COPD, and hypertension. After the Sleep Visit, participants were contacted every 4 months; $>97 \%$ of contacts were completed. Reported deaths were confirmed with death certificates and medical records. CVD mortality was based on the underlying cause of death as determined by a study physician adjudicator. Cause of death was broadly categorized by ICD-9 codes as CVD (codes 396.9-442, 966.71, 785.51), cancer (codes 141.9-208.0), and other causes (reported codes not in previous categories).

\section{The Sleep Heart Health Study}

The second study was the Sleep Heart Health Study (SHHS), a community-based, prospective cohort study designed to examine the CVD consequences of sleep-disordered breathing. Details of the study design have been reported previously. ${ }^{26,28,29}$ A total of 6441 men and women aged 40 years or older completed the baseline examination (1995-1998), including a Sleep Habits Questionnaire, anthropometric information, and overnight unattended PSG. Institutional Review Board approval was obtained at all participating institutions, and all participants signed informed consent. Of the 6441 participants enrolled in SHHS, 637 withdrew their consent due to sovereignty issues (Strong Heart Study participants are not included in the shared SHHS data). Therefore, our dataset includes the remaining 5804 participants.

Concurrent CVD disease was defined as a history of physiciandiagnosed angina, heart failure, myocardial infarction, stroke, or coronary revascularization, and it was determined by adjudicated surveillance data provided by the parent cohorts or by self-report at enrolment. Questionnaires were administered to determine participant demographics, alcohol use, and smoking history. The details of the sleep study have been published previously. ${ }^{29}$ All PSGs were scored at the same central sleep reading centre as was the MrOS Study (Case Western Reserve University, directed by S Redline) using similar methods other than reliance on thermistry and inductance plethysmography (SHHS) rather than nasal pressure, thermistry, and inductance plethysmography (MrOS) for 

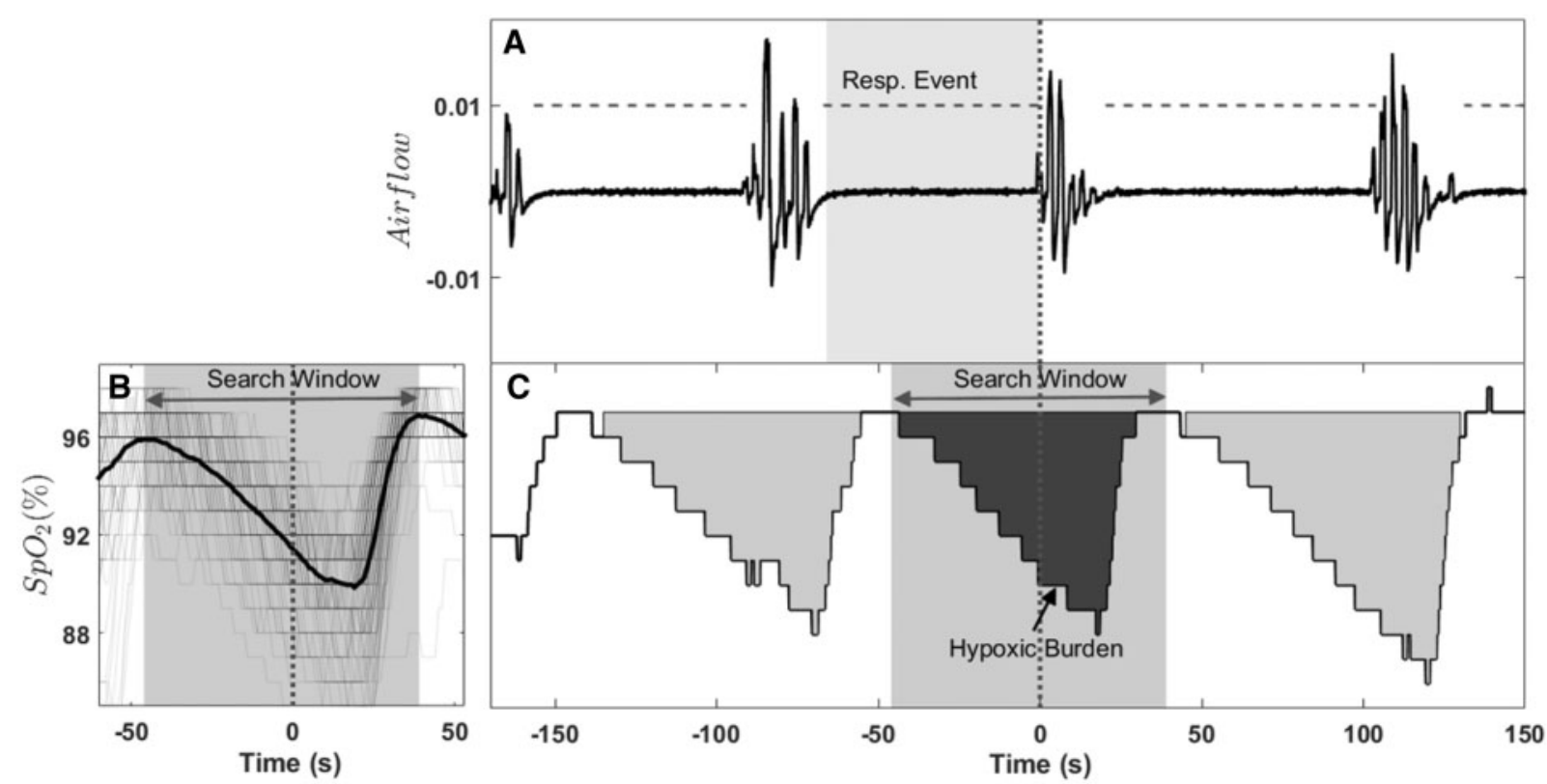

Figure I Example of hypoxic burden calculation for an individual respiratory event. (A) The nasal cannula airflow and annotated respiratory events is shown. (B) The overlaid oxygen saturation signals $\left(\mathrm{SpO}_{2}\right)$ associated with all respiratory events for one individual is shown. These signals were synchronized at the termination of respiratory events (time zero) and averaged to calculate the search window (the time between two peaks). The search window was used to calculate the hypoxic burden for individual events. That is, the area under saturation curve within the search window ( $C$ ).

The total hypoxic burden was defined as the sum of individual burdens divided by total sleep time. Resp. Event, respiratory event.

identification of respiratory events. Events were similarly identified on the basis of amplitude reduction only, with $\mathrm{AHI}$ derived after further linking hypopnoeas to desaturations of $\geq 3 \%$ or arousals. $\mathrm{SpO}_{2}$ signals were captured by fingertip pulse oximeter (Nonin, Minneapolis, MN, USA) sampled at $1 \mathrm{~Hz}$.

Death from any cause (including CVD death), the primary endpoint for this report, was identified and confirmed for SHHS using follow-up interviews, written annual questionnaires, or telephone contacts with study participants or next-of-kin, surveillance of local hospital records and community obituaries, and linkage with the Social Security Administration Death Master File. ${ }^{19}$

\section{Nocturnal hypoxic burden as a measure of obstructive sleep apnoea severity}

The total area under the ventilation curve, using eupneic ventilation as the baseline, can be used to quantify the total ventilatory deficit caused by obstruction in the airway. However, due to the differences in airflow measurement methodologies (e.g. nasal pressure airflow, thermal airflow, respiratory belts ${ }^{31}$ ) and lack of standardization across clinical and cohort studies, pulse oximetry may be used as a readily available surrogate of ventilation. Therefore, instead of the area under the ventilation curve, the area under the desaturation curve associated with respiratory events was used to quantify sleep apnoea severity. We derived a measure of sleeprelated 'hypoxic burden' aimed at capturing the total amount of respiratory event-related hypoxaemia over the sleep period. The hypoxic burden was defined as the total area under the respiratory event-related desaturation curve. For each individually identified apnoea or hypopnoea (obstructive and central regardless of associated desaturation), the preevent baseline saturation was defined as the maximum $\mathrm{SpO}_{2}$ during the $100 \mathrm{~s}$ prior to the end of the event. The area under this baseline value was calculated over a subject-specific search window (Figure 1) for each event. For a robust area calculation (particularly for the events without $\mathrm{SpO}_{2}$ recovery to the baseline value), the subject-specific search window was obtained from an averaged desaturation curve. The average desaturation curve for each participant was determined by overlaying $\mathrm{SpO}_{2}$ signals with respect to the end of events (Figure 1, see Supplementary material online for more details). The hypoxic burden was then obtained by adding these individual desaturation areas and dividing the total area by the sleep duration, with the units of hypoxic burden being $(\% \mathrm{~min}) / \mathrm{h}$. For example, a hypoxic burden of $20(\% \mathrm{~min}) / \mathrm{h}$ is equivalent to $20 \mathrm{~min}$ of $1 \%$ desaturation per hour or $5 \mathrm{~min}$ of $4 \%$ desaturation per hour (see Supplementary material online for more details).

\section{Statistical analysis}

Distributions of covariates and sleep measures were summarized by mortality status. A series of Cox proportional models were constructed to assess the association between CVD and all-cause mortality risks and hypoxic burden. To choose the functional form of the hypoxic burden variable in the Cox models that best describes the association between hypoxic burden and our outcome, the martingale residuals of the unadjusted Cox model were plotted for the untransformed, logtransformed, square root-transformed, and power two-transformed hypoxic burden. The fitted lines with Lowess function revealed linearity in the log-transformed hypoxic burden (see Supplementary material online for more details on the Cox regression analysis of log-transformed hypoxic burden). For help with interpretation without making any assumptions regarding linearity, the hypoxic burden was also categorized based on its quintiles: $<20$ th percentile (reference), 20th-40th percentile, 40th60 th percentile, 60 th-80th percentile, and $>80$ th percentile. 
Covariates were chosen based on established clinical relationships to mortality. The models were adjusted for demographic/anthropometric covariates and pre-existing medical conditions. Covariates used for both MrOS and SHHS included age, body mass index (BMI), race, smoking status (current, former, or never), concurrent medical conditions such as hypertension, diabetes, stroke, COPD, and lipid-lowering medication (Statin) use, with each defined as previously described. ${ }^{18,22}$ To test the association between hypoxic burden and mortality and whether the association persisted after covariate adjustment, several models were considered. Model 0 included only the hypoxic burden index. In Model 1, demographic/anthropometric covariates [age, BMI, race and gender (not applicable in MrOS), race)], total sleep time (TST, $\leq 5,5-8,>8 \mathrm{~h}$ ), study site, smoking status, alcohol use $(<1,1-13,>14$ glasses/week; only available in MrOS due to $>400$ missing values in SHHS), and non-CVD-related medical conditions, including COPD and renal failure (not available in SHHS). Model 2a, which included covariates in Model 1 and $\mathrm{AHI}$, was used to test the association for hypoxic burden after adjusting for the AHI as the commonly used measure of sleep apnoea severity. Due to the presence of collinearity between $\mathrm{AHI}$ and oxygen desaturation index (ODI), Model $2 \mathrm{~b}$ replaced the $\mathrm{AHI}$ with ODI in Model $2 \mathrm{a}$ and assessed the association between hypoxic burden and CVD mortality. Model 3 added commonly-used measures of overnight hypoxaemia such as percent sleep time with $\mathrm{SpO}_{2}<90 \%$ (TST90) and average event-related minimum saturation (MinSat) to Model 2a. Finally, Model 4 was constructed by additionally adjusting Model 3 for concurrent cardiometabolic conditions (fully adjusted model). In MrOS, concurrent CVD included coronary heart disease, peripheral vascular disease, claudication, myocardial infarction, angina, transient ischaemic attack, and heart failure. In SHHS, concurrent CVD included angina, heart failure, myocardial infarction, stroke, and coronary revascularization. ${ }^{19} \mathrm{AHI}$ and $\mathrm{ODI}$ were modelled as continuous variables and the risk was reported for every 15 events/h increase in the $\mathrm{AHI}$ or the ODI. TST90 was modelled as a continuous variable and the risk was reported for every $10 \%$ increase in the TST90. Modelling the AHI as a categorical variable with several clinical cut-points (5, 15, and 30 events/ h) or quartiles did not alter the results; therefore, it was kept as a continuous variable. To avoid multicollinearity, it was ensured that the variance inflation factors remained below five. ${ }^{32}$ To plot the adjusted survival curves for the hypoxic burden quintiles in MrOS, Cox regression Model 4 was used to obtain the predicted survival curve for every participant in MrOS. These individual survival curves were then averaged to obtain the adjusted survival curves. ${ }^{33}$ Statistical analyses were conducted using the $\mathrm{R}$ statistical package (http://www.r-project.org (4 October 2018)).

\section{Results}

The characteristics of participants in MrOS and SHHS studies, grouped by their mortality status, are shown in Tables 1 and 2, respectively.

\section{The Osteoporotic Fractures in Men Study}

Of the 2907 available PSG files, 42 had pending documentation to verify the death, 11 had poor quality SpO2 signals, and 111 had incomplete covariate data, resulting in 2743 participants in the analytical dataset. Over an average of $10.0 \pm 3.5$ years, a total of 440 CVD-related deaths and 1270 all-cause deaths were identified. The characteristics of participants grouped by their mortality status are shown in Table 1.

The results of the Cox regression models are shown in Table 3. The unadjusted hazard ratio of CVD mortality for second, third, fourth, and fifth quintile were 1.24 [95\% confidence interval $(\mathrm{Cl}) 0.89-$ 1.72], 1.10 (95\% Cl 0.78-1.54), 1.64 (95\% Cl 1.20-2.24), and 2.31 (95\% Cl 1.71-3.12), respectively (Table 3, Model 0). After adjusting for anthropometric/demographic variables, smoking, alcohol and, in MrOS, COPD and renal failure, the hazard ratio was somewhat attenuated but remained significant for the fourth and fifth quintiles [1.44 (95\% Cl 1.05-1.97) and $1.75(95 \% \mathrm{Cl} 1.28,2.40)$, Table 3]. The association of hypoxic burden fourth and fifth quintiles and CVD mortality persisted and remained significant after further adjustment for $\mathrm{AHI}$ in Model 2a $\left[\mathrm{HR}\left(\mathrm{Q}_{4}\right)=1.77(95 \% \mathrm{Cl} 1.23-2.54)\right), \mathrm{HR}\left(\mathrm{Q}_{5}\right)=2.61(95 \%$ Cl 1.65-4.14)] or ODI in Model 2b $\left[\mathrm{HR}\left(\mathrm{Q}_{4}\right)=1.57(95 \% \mathrm{Cl} 1.09-\right.$ 2.26)), $\left.\mathrm{HR}\left(\mathrm{Q}_{5}\right)=2.10(95 \% \mathrm{Cl} 1.31-3.34)\right]$. Further adjustments for alternative measurements of hypoxaemia (MinSat and TST90, Model 3; Table 3) or concurrent CVD (Model 4; Table 3, Supplementary material online, Table S1) did not appreciably change the hazard ratios for hypoxic burden. Figure 2 displays the adjusted survival curves for the Cox regression model 4. The online supplementary material (Table S3) further shows the hazard ratios when modelling log-transformed hypoxic burden. In addition to CVD mortality, a significant association between hypoxic burden and all cause mortality was observed (see Supplementary material online, Table S4).

\section{Sleep Heart Health Study}

From 6441 participants enrolled in SHHS, 5804 studies were available in the National Sleep Research Resource. After excluding 32 studies with missing or poor-quality $\mathrm{SpO}_{2}$ signals, 752 with missing CVD mortality status, and 348 with incomplete covariate data, 4672 subjects were available for CVD mortality. Over an average of $10.9 \pm 3.1$ years, a total of 313 CVD-related deaths were observed in these individuals (censoring date of 7 December $2011^{34}$ ). A total of 5111 individuals with complete covariates were available for all-cause mortality (1142 all-cause deaths). The characteristics of these participants, grouped by their mortality status, are shown in Table 2.

Similar to MrOS, unadjusted (Model 0), partially adjusted (Models 1, 2a, 2b, 3), and fully adjusted (Model 4) Cox regression models showed a significant hazard ratio of CVD mortality for the fifth quintile of hypoxic burden (Table 3). In addition, unadjusted model and partially adjusted Models 2a, 2b, and 3 showed a significant hazard ratio of CVD mortality for the fourth quintile of hypoxic burden (Table 3). The upper $20 \%$ of hypoxic burden had fully adjusted hazard ratios of 1.96 (95\% Cl 1.11-3.43) in Model 4 (Table 3). No significant association between hypoxic burden and all-cause mortality was observed in SHHS study (see Supplementary material online, Table S5).

\section{Associations between hypoxic burden and conventional polysomnographic parameters}

In both MrOS and SHHS, the hypoxic burden was variably associated with other PSG parameters, such as AHI, ODI, arousal index, wake time after sleep onset, and TST90. These associations weakened for patients with moderate to severe sleep apnoea (see Supplementary material online, Figure $S 2$ for the correlation analysis). For example, in MrOS, for patients with moderate to severe sleep apnoea, the correlation between the hypoxic burden and PSG parameters such as AHI, TST90, and ODI were 0.70, 0.49, and 0.74, respectively (see Supplementary material online, Figure S2). 
Table I Sample characteristics by all-cause and cardiovascular mortality in MrOS

\begin{tabular}{|c|c|c|c|}
\hline & Alive $(N=1473)$ & $\begin{array}{l}\text { All-cause mortality } \\
(N=1270)\end{array}$ & $\begin{array}{l}\text { CVD mortality } \\
(N=440)\end{array}$ \\
\hline Age (years), mean (SD) & $74.3(4.5)$ & $78.8(5.5)$ & $79.2(5.5)$ \\
\hline $\mathrm{BMI}\left(\mathrm{kg} / \mathrm{m}^{2}\right)$ & $27.2(3.6)$ & $27.1(4.1)$ & $27.3(3.9)$ \\
\hline \multicolumn{4}{|l|}{ Race, $n(\%)$} \\
\hline Caucasian & $1312(89.1)$ & $1177(92.7)$ & $411(93.4)$ \\
\hline African American & $54(3.7)$ & $38(3.0)$ & $8(1.8)$ \\
\hline Other race & $107(7.3)$ & $55(4.3)$ & $21(4.8)$ \\
\hline \multicolumn{4}{|l|}{ Alcohol, n (\%) } \\
\hline$<1$ glasses/week & $660(44.8)$ & $608(47.9)$ & $218(49.5)$ \\
\hline 1-13 glasses/week & $725(49.2)$ & $601(47.3)$ & $202(45.9)$ \\
\hline $14+$ glasses/week & $88(6.0)$ & $61(4.8)$ & $20(4.6)$ \\
\hline Congestive heart failure, $n(\%)$ & $49(3.3)$ & $111(8.7)$ & $58(13.2)$ \\
\hline Cardiovascular disease (including congestive heart failure), $n$ (\%) & $459(31.2)$ & $636(50.1)$ & $260(59.1)$ \\
\hline Hypertension, $n(\%)$ & $658(44.7)$ & $703(55.4)$ & $272(61.8)$ \\
\hline Stroke, $n(\%)$ & $34(2.3)$ & $68(5.4)$ & $27(6.1)$ \\
\hline Diabetes, $n(\%)$ & $159(10.8)$ & $196(15.4)$ & $84(19.1)$ \\
\hline Renal failure, $n(\%)$ & $5(0.3)$ & $21(1.7)$ & $7(1.6)$ \\
\hline Chronic obstructive pulmonary disease, $n(\%)$ & $60(4.1)$ & $82(6.5)$ & $19(4.3)$ \\
\hline \multicolumn{4}{|l|}{ Smoking status, $n$ (\%) } \\
\hline Never & $613(41.6)$ & $484(38.1)$ & $164(37.3)$ \\
\hline Former & $836(56.8)$ & $758(59.7)$ & $271(61.6)$ \\
\hline Current & $24(1.6)$ & $28(2.2)$ & $5(1.1)$ \\
\hline Statin use, $n(\%)$ & $631(42.8)$ & $499(39.3)$ & $196(44.5)$ \\
\hline \multicolumn{4}{|l|}{ Total sleep time (TST), $n$ (\%) } \\
\hline$>5-8 \mathrm{~h}$ & $1221(82.9)$ & $970(76.4)$ & $336(76.4)$ \\
\hline$\leq 5 \mathrm{~h}$ & $224(15.2)$ & $257(20.2)$ & $92(20.9)$ \\
\hline$>8 \mathrm{~h}$ & $28(1.9)$ & $43(3.4)$ & $12(2.7)$ \\
\hline Apnoea-hypopnoea index (events/h) & $15.7(13.9)$ & $17.8(14.8)$ & $18.9(15.1)$ \\
\hline TST90 (\%TST) & $3.23(7.76)$ & $5.11(10.9)$ & $5.36(10.4)$ \\
\hline Hypoxic burden (\%min/h) & $53.3(47.8)$ & $64.3(57.7)$ & $72.7(61.5)$ \\
\hline Q1 ( $\leq 20 \% \min / h), n(\%)$ & $324(22.0)$ & $225(17.7)$ & 64 (14.5) \\
\hline Q2 (20-34 \%min/h), n (\%) & $304(20.6)$ & $248(19.5)$ & $77(17.5)$ \\
\hline Q3 (34-53 \%min/h), n (\%) & $324(22.0)$ & $224(17.6)$ & $71(16.1)$ \\
\hline Q4 (53-88 \%min/h), n (\%) & $283(19.2)$ & $270(21.3)$ & $100(22.7)$ \\
\hline Q5 (>88 \%min/h), n (\%) & $238(16.2)$ & $303(23.9)$ & $128(29.1)$ \\
\hline
\end{tabular}

\section{Impact of desaturation depth on the association of hypoxic burden and cardiovascular mortality}

To further investigate whether the impact of short and deep desaturations on CVD mortality hazard ratio is different than long and shallow ones, we performed additional analyses in the MrOS cohort by transforming event-associated raw desaturations using the squareroot (to diminish the effect of desaturation depth) and square (to exaggerate the effect of desaturation depth, Supplementary material online, Figure S4). The standardized adjusted hazard ratios in Model 2 a remained similar for square root-transformed, untransformed, and square-transformed hypoxic burden, respectively (see Supplementary material online, Table S7). These findings suggest that, at least in MrOS cohort, the short and deep desaturations were similar to long and shallow desaturations in terms of their impact on the observed CVD mortality hazard ratios.

\section{Discussion}

Using two large samples of middle-aged and older adults from several communities in the USA, we found that OSA severity quantified as the respiratory event-associated hypoxic burden was independently associated with CVD mortality. This association persisted after adjusting for a large number of potential confounders and possible mediators, including prevalent CVD as well as AHI, TST90, and sleep duration. In contrast, CVD mortality was not associated with $\mathrm{AHI}$ when it was assessed as an independent predictor. When examining other measurements of hypoxaemia, TST90 was significantly associated with CVD mortality in SHHS and not in MrOS, while the ODI was associated with CVD mortality in MrOS but not SHHS. Therefore, these data suggest that CVD mortality is strongly associated with a quantitative measure of hypoxic burden associated with respiratory disturbances, and that this measure significantly and more consistently (across cohorts) predicts this outcome than a simpler 
Table 2 Sample characteristics by all-cause and cardiovascular mortality in SHHS

\begin{tabular}{|c|c|c|c|}
\hline & Alive $(N=3969)$ & $\begin{array}{l}\text { All-cause mortality } \\
(N=1142)\end{array}$ & $\begin{array}{l}\text { CVD mortality } \\
(N=313)\end{array}$ \\
\hline Age (years), mean (SD) & $61.0(9.9)$ & $72.8(9.1)$ & $75.6(7.7)$ \\
\hline \multicolumn{4}{|l|}{ Gender, $n(\%)$} \\
\hline Male & $1796(45.3)$ & $615(53.9)$ & $168(53.7)$ \\
\hline Female & $2173(54.7)$ & $527(46.1)$ & $145(46.3)$ \\
\hline $\mathrm{BMI}\left(\mathrm{kg} / \mathrm{m}^{2}\right)$ & $28.3(5.1)$ & $27.7(5.0)$ & $27.4(4.8)$ \\
\hline \multicolumn{4}{|l|}{ Race, $n(\%)$} \\
\hline Caucasian & $3445(86.8)$ & $1016(89.0)$ & $277(88.5)$ \\
\hline Non-Caucasian & $524(13.2)$ & $126(11.0)$ & $36(11.5)$ \\
\hline Diabetes, $n(\%)$ & $193(4.9)$ & $169(14.8)$ & $66(21.1)$ \\
\hline Hypertension, $n(\%)$ & $1479(37.3)$ & $692(60.6)$ & $218(69.6)$ \\
\hline Stroke, $n(\%)$ & $89(2.2)$ & $80(7.0)$ & $32(10.2)$ \\
\hline Chronic obstructive pulmonary disease, $n(\%)$ & $33(0.8)$ & $24(2.1)$ & $3(0.1)$ \\
\hline \multicolumn{4}{|l|}{ Smoking, $n(\%)$} \\
\hline Never & $1946(49.0)$ & $476(41.7)$ & $143(45.7)$ \\
\hline Former & $354(8.9)$ & $111(9.7)$ & $21(6.7)$ \\
\hline Current & $1669(42.1)$ & $555(48.6)$ & $149(47.6)$ \\
\hline Cardiovascular disease (including congestive heart failure), $n$ (\%) & $347(8.7)$ & $288(25.2)$ & $110(35.1)$ \\
\hline Lipid-lowering medication use, $n(\%)$ & $462(11.6)$ & $149(13.0)$ & $49(15.7)$ \\
\hline \multicolumn{4}{|l|}{ Total sleep time (TST), $n(\%)$} \\
\hline $5-8 \mathrm{~h}$ & $493(12.4)$ & $206(18.0)$ & $58(18.5)$ \\
\hline$\leq 5 \mathrm{~h}$ & $19(0.5)$ & $22(1.9)$ & $7(2.2)$ \\
\hline$>8 \mathrm{~h}$ & $3457(87.1)$ & $914(80.0)$ & $248(79.2)$ \\
\hline Apnoea-hypopnoea index (events/h) & $17.1(15.3)$ & $20.9(17.4)$ & $20.9(15.9)$ \\
\hline TST90 (\%TST) & $2.73(8.5)$ & $6.32(15.2)$ & $6.47(16.5)$ \\
\hline Hypoxic burden (\% min/h) & $46.6(53.8)$ & $62.6(66.5)$ & $64.3(63.0)$ \\
\hline Q1 (s16\%min/h), n (\%) & $857(21.6)$ & $155(13.6)$ & $29(9.27)$ \\
\hline Q2 (16-28 \% min/h), n (\%) & $851(21.4)$ & $172(15.1)$ & $48(15.3)$ \\
\hline Q3 (28-43 \% min/h), n (\%) & $818(20.6)$ & $213(18.7)$ & $65(20.8)$ \\
\hline Q4 (43-71 \% min/h), n (\%) & $754(19.0)$ & $270(23.6)$ & $74(23.6)$ \\
\hline Q5 (>71 \%min/h), n (\%) & $689(17.4)$ & $332(29.1)$ & $97(31.0)$ \\
\hline
\end{tabular}

measure of event frequency (AHI), overall hypoxaemia (TST90), or the desaturation frequency (ODI) during sleep.

\section{Obstructive sleep apnoea-related hypoxaemia and risk of cardiovascular disease}

Intermittent hypoxaemia associated with individual hypopnoeas and apnoeas has been shown to be a key factor in mediating CVD risk ${ }^{35}$ in OSA. Previous studies have shown the associations between intermittent hypoxaemia and indices of subclinical CVD or CVD risk factors, including endothelial dysfunction, ${ }^{36}$ increased sympathetic activation, ${ }^{37}$ inflammation, ${ }^{38}$ oxidative stress, ${ }^{39}$ and metabolic dysfunction. ${ }^{40}$ Measurements of hypoxaemia also have been shown to predict incident atrial fibrillation, ${ }^{41}$ sudden cardiac death, ${ }^{42}$ and mortality in stable heart failure patients. ${ }^{21}$ However, there is not a consensus on which measurement(s) of overnight oxygen saturation are most sensitive or predictive of incident CVD. For example, in a relatively small study of middle-aged men, Peker et al. ${ }^{20}$ reported that the ODI associated with an increased risk of incident CVD in middleaged men. Total sleep time with an oxygen saturation below $90 \%$ or
80\% (TST90, TST80), although commonly reported in sleep studies, has not been shown to be a consistent predictor of mortality in patients with OSA. ${ }^{19,22}$ For example, Punjabi et al. ${ }^{19}$ found an association between all-cause mortality and prolonged oxygen desaturation (TST90 > 2.70\% total sleep time) in younger but not older men in SHHS. In contrast, Smagula et al. ${ }^{22}$ found a significant association between all-cause mortality and severe, prolonged oxygen desaturation (TST80 $\geq 1 \%$ total sleep time) in older men (MrOS cohort). One challenge of some commonly used metrics is that they depend on somewhat arbitrary absolute thresholds (e.g. $90 \%$ and $80 \%$ ), which may not apply across populations. Metrics such as TST90 also are not specific to the individual apnoeas and hypopnoeas but may reflect underlying lung disease or a hypoventilation disorder, and thus are less specific markers of OSA-related hypoxic stress compared with the hypoxic burden measure. TST90 also does not provide direct information on the targets for OSA treatment (e.g. CPAP), which mostly address episodic airway obstruction and its ensuing hypoxaemia. In contrast, the hypoxic burden index quantifies a target for CPAP, and may possibly better identify individuals who will respond to CPAP than less specific measures of hypoxaemia or the AHI, which does not measure the associated physiological stress. 
Table 3 Hypoxic burden predicts cardiovascular-related mortality in MrOS and SHHS

\begin{tabular}{|c|c|c|c|}
\hline Covariates & $\begin{array}{l}\text { Hypoxic burden } \\
\text { quintiles }\end{array}$ & $\begin{array}{l}\text { MrOS } \\
\text { HR }(95 \% \mathrm{Cl})\end{array}$ & $\begin{array}{l}\text { SHHS } \\
\text { HR }(95 \% \mathrm{Cl})\end{array}$ \\
\hline \multirow[t]{5}{*}{ Model 0: none } & $\mathrm{Q}_{1}$ & 1.00 & 1.00 \\
\hline & $\mathrm{Q}_{2}$ & $1.24(0.89,1.72)$ & $1.67(1.06-2.65)^{*}$ \\
\hline & $\mathrm{Q}_{3}$ & $1.10(0.78,1.54)$ & $2.30(1.49-3.57)^{* * * *}$ \\
\hline & $\mathrm{Q}_{4}$ & $1.64(1.20,2.24)^{* *}$ & $2.71(1.76-4.16)^{* * * *}$ \\
\hline & $Q_{5}$ & $2.31(1.71,3.12)^{* * * *}$ & $3.88(2.56-5.88)^{* * * *}$ \\
\hline \multirow{5}{*}{$\begin{array}{l}\text { Model 1: anthropometric }{ }^{\mathrm{a}} \text {, sleep duration, } \\
\text { smoking, alcohol }^{\mathrm{b}} \text {, non-CVD medical history }\end{array}$} & $\mathrm{Q}_{1}$ & 1.00 & 1.00 \\
\hline & $\mathrm{Q}_{2}$ & $1.22(0.87,1.70)$ & $1.41(0.88-2.24)$ \\
\hline & $\mathrm{Q}_{3}$ & $1.12(0.80,1.58)$ & $1.25(0.80-1.96)$ \\
\hline & $\mathrm{Q}_{4}$ & $1.44(1.05,1.97)^{*}$ & $1.51(0.97-2.35)$ \\
\hline & $Q_{5}$ & $1.75(1.28,2.40)^{* * *}$ & $1.62(1.04-2.51)^{*}$ \\
\hline \multirow[t]{5}{*}{ Model 2a: Model 1 + AHI } & $\mathrm{Q}_{1}$ & 1.00 & 1.00 \\
\hline & $\mathrm{Q}_{2}$ & $1.29(0.92,1.81)$ & $1.45(0.91-2.31)$ \\
\hline & $\mathrm{Q}_{3}$ & $1.26(0.88,1.79)$ & $1.34(0.85-2.12)$ \\
\hline & $\mathrm{Q}_{4}$ & $1.77(1.23,2.54)^{* * *}$ & $1.69(1.05-2.70)^{*}$ \\
\hline & $Q_{5}$ & $2.61(1.65,4.14)^{* * *}$ & $2.03(1.17-3.50)^{*}$ \\
\hline \multirow[t]{5}{*}{ Model 2b: Model 1 + ODI } & $\mathrm{Q}_{1}$ & 1.00 & 1.00 \\
\hline & $\mathrm{Q}_{2}$ & $1.25(0.89,1.75)$ & $1.43(0.90-2.28)$ \\
\hline & $\mathrm{Q}_{3}$ & $1.18(0.83,1.68)$ & $1.31(0.83-2.06)$ \\
\hline & $\mathrm{Q}_{4}$ & $1.57(1.09,2.26)^{*}$ & $1.63(1.04-2.57)^{*}$ \\
\hline & $\mathrm{Q}_{5}$ & $2.10(1.31,3.34)^{* *}$ & $1.92(1.16-3.15)^{*}$ \\
\hline \multirow[t]{5}{*}{ Model 3: Model 2a + MinSat + TST90 } & $\mathrm{Q}_{1}$ & 1.00 & 1.00 \\
\hline & $Q_{2}$ & $1.30(0.93,1.82)$ & $1.50(0.94-2.39)$ \\
\hline & $\mathrm{Q}_{3}$ & $1.29(0.91,1.85)$ & $1.41(0.89-2.25)$ \\
\hline & $\mathrm{Q}_{4}$ & $1.83(1.27,2.64)^{* *}$ & $1.78(1.10-2.86)^{*}$ \\
\hline & $Q_{5}$ & $2.77(1.73,4.42)^{* * * *}$ & $2.09(1.20-3.63)^{* *}$ \\
\hline \multirow[t]{5}{*}{ Model 4: Model $3+$ cardio-metabolic diseases $^{\mathrm{d}}$} & $\mathrm{Q}_{1}$ & 1.00 & 1.00 \\
\hline & $\mathrm{Q}_{2}$ & $1.19(0.85,1.68)$ & $1.48(0.93-2.37)$ \\
\hline & $\mathrm{Q}_{3}$ & $1.26(0.88,1.81)$ & $1.34(0.85-2.14)$ \\
\hline & $\mathrm{Q}_{4}$ & $1.81(1.25,2.62)^{* *}$ & $1.61(1.00-2.61)$ \\
\hline & $\mathrm{Q}_{5}$ & $2.73(1.71,4.36)^{* * *}$ & $1.96(1.11-3.43)^{*}$ \\
\hline
\end{tabular}

Unadjusted and adjusted hazard ratios for hypoxic burden in different models.

AHI, apnoea-hypopnoea index (3\% criterion); MinSat, event-associated minimum saturation; ODI, oxygen desaturation index ( $\geq 3 \%$ desaturations); TST90, percent time spent below oxygen saturation of $90 \%$.

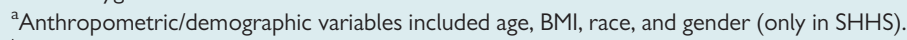

${ }^{\mathrm{b}}$ The number of missing values for alcohol consumption was substantially large in SHHS, therefore, it was only available in MrOS.

${ }^{c}$ Non-CVD medical history included chronic obstructive pulmonary disease (COPD) and renal failure (only available in MrOS).

${ }^{d}$ Concurrent cardio-metabolic disease included hypertension, diabetes, stroke, congestive heart failure, concurrent cardiovascular disease (MrOS: coronary heart disease, peripheral vascular disease, claudication, myocardial infarction, angina, and transient ischaemic attack; SHHS: angina, myocardial infarction, and coronary revascularization), and lipid-lowering medication use.

$* P<0.05$; ** $P<0.01$; *** $P<0.001$.

\section{All-cause mortality}

While an association was found between hypoxic burden and CVD mortality in both cohorts, a significant association for all-cause mortality was only found in MrOS. A weaker and less-consistent association between hypoxic burden and all-cause mortality suggests that there may be a distinct biological pathway linking intermittent hypoxia associated with respiratory events to CVD death.

\section{Apnoea-hypopnoea index as a predictor of obstructive sleep apnoea outcomes}

Several studies in clinical and community populations have shown associations between sleep-disordered breathing and mortality risk. ${ }^{10,17,19,21-23}$ However, the results are conflicting and vary according to the metrics used to describe OSA and the population studied. For instance, a prospective analysis of SHHS revealed an association between all-cause mortality and severe OSA (AHI > 30, all apnoeas and hypopnoeas with $\geq 4 \%$ desaturation), yet the results were only significant in men aged $40-70$ years. ${ }^{19}$ Consistent with their findings, after adjusting for confounders, we did not find a significant association between AHI and CVD and all-cause mortality in the MrOS cohort, which was comprised of men aged 65 years and older. This finding was irrespective of whether AHI was modelled as a continuous variable or a categorical variable using clinical cut-points. One reason why $\mathrm{AHI}$ is not a strong predictor of mortality could be that it 


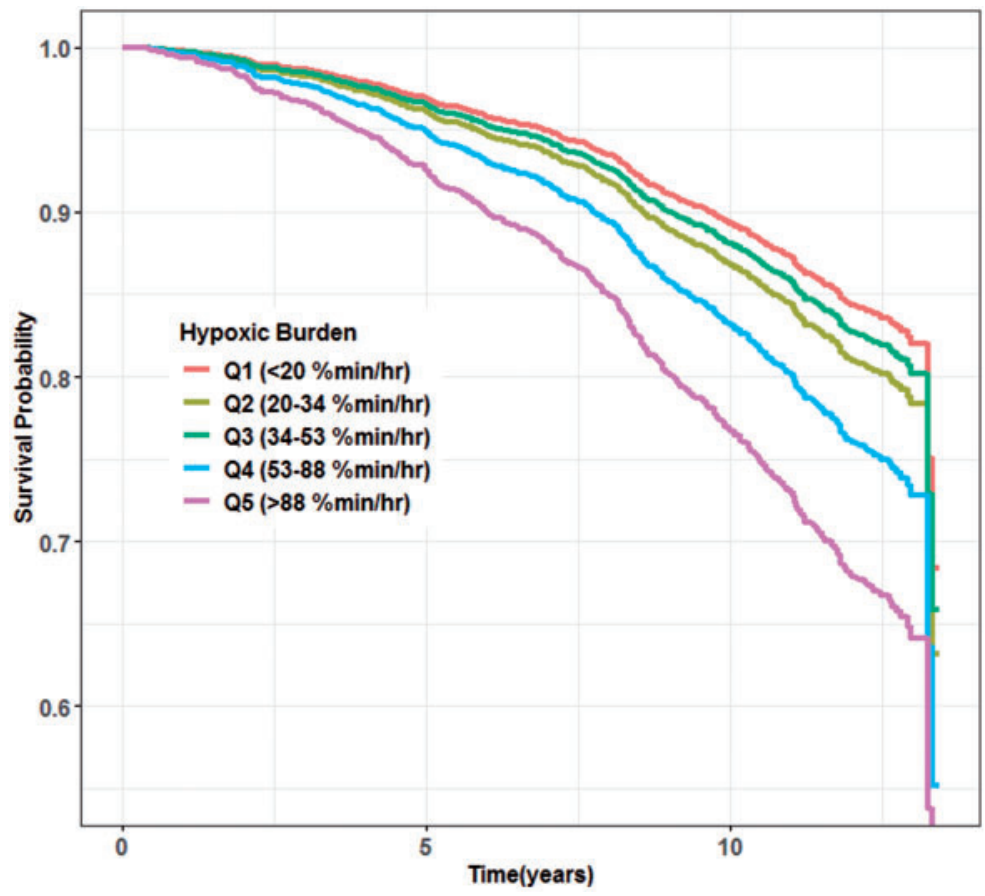

Figure 2 Adjusted survival curves for cardiovascular mortality across categories of the hypoxic burden in MrOS. These curves were obtained from Model 4. The adjusted survival curves were obtained by averaging the predicted survival curves for every observation in MrOS study.

only measures the frequency of respiratory events and has no information about the duration and severity of blood gas changes. For example, for an $\mathrm{AHI}$ of 40 events/h, the hypoxic burden ranged from 40 to $300 \% \mathrm{~min} / \mathrm{h}$. This broad range is due to the variability in the depth and the duration of event-related oxygen desaturations that is not captured by the AHI (see Supplementary material online, Figure S3). In addition, $\mathrm{AHI}$ is also subject to a number of measurement challenges, including the accuracy of identifying apnoeas and hypopnoeas and the potential misclassification of disease severity by combining apnoeas and hypopnoeas. ${ }^{43}$ On the other hand, the hypoxic burden incorporates the depth and the duration of respiratory-event-related hypoxaemia in addition to their event frequency and does not depend on the choice of desaturation cut-offs for identifying events. Furthermore, hypoxic burden can be derived without electroencephalography-based measurements of arousal, and thus could be extracted from home sleep apnoea tests, which are increasingly used clinically, potentially providing a useful means for improving characterization of CVD risk in patients with OSA.

Several recent large trials (Sleep Apnea Cardiovascular Endpoints $(\mathrm{SAVE})^{15}$ and the Treatment of Predominant Central Sleep Apnea by Adaptive Servo Ventilation in Patients With Heart Failure (SERVE$\mathrm{HF})^{44}$ ) have failed to find a positive long-term CVD or all-cause mortality benefit of positive airway treatment. ${ }^{15,44}$ A number of reasons have been suggested for these findings, ${ }^{45}$ including the possibility of inadequate case selection. By selecting participants on the basis of $\mathrm{AHI}$ or ODI may have not well identified those individuals at highest mortality risk. Secondary analyses of data from these trials, reclassifying participants based on severity of hypoxic burden, may help clarify whether individuals with greater hypoxic burden may benefit more from positive pressure treatment than those with less hypoxic burden.

\section{Strengths and limitations}

There were a number of notable strengths of this study, including the automated generation of an index of hypoxic burden that only required data from oximetry channel and respiratory events-data readily available from home sleep apnoea tests. The findings were robust to multiple covariate adjustments and were consistent across two independent samples that differed by demographics, suggesting likely generalizability of the results. However, the study had several limitations, including the under-representation of minority populations and young individuals. The age representation and sample size did not permit assessment in women alone or in younger. Given the observational nature of these studies, residual confounding cannot be excluded.

It is important to note that despite the advantages of a single measure of hypoxic burden that captures the depth and duration of respiratory-related desaturation, it is possible that alternative metrics that characterize other patterns of hypoxic stress, may prove to be useful. For example, our measure does not distinguish short and deep desaturations from and long and shallow ones. For example, $20 \mathrm{~min}$ of $1 \%$ desaturation per hour and $5 \mathrm{~min}$ of $4 \%$ desaturation per hour would be similarly represented. However, we conducted exploratory analyses (see Supplementary material online, Table S7 and Figure S4) that suggest that the observed hazard ratios were consistent even when the contribution of deeper desaturations were mathematically varied. It is also important to recognize that our outcomes were focused on CVD and all-cause mortality, and it is possible that 
alternative metrics, such as arousal intensity ${ }^{46-48}$ and odds ratio product $^{49}$ that capture sleep fragmentation, may be important in assessing outcomes such as sleepiness, cognition, and quality of life.

\section{Conclusions}

We developed an OSA severity measure, the hypoxic burden, which we believe better characterizes the underlying pathology of OSA and is associated with an increased risk of CVD mortality independent of the $\mathrm{AHI}$ and multiple confounding variables. These data show that hypoxic burden appears to be a better measure of OSA severity with respect to the CVD consequences of disordered breathing. Given the increasing prevalence of OSA in the general population, future studies are necessary to determine whether this metric can be used to predict the extent of which different OSA treatments can prevent non-fatal and fatal CVD events.

\section{Supplementary material}

Supplementary material is available at European Heart Journal online.

\section{Funding}

The Osteoporotic Fractures in Men (MrOS) Study is supported by National Institutes of Health funding. The following institutes provide support: the National Institute of Arthritis and Musculoskeletal and Skin Diseases (NIAMS), the National Institute on Aging (NIA), the National Center for Research Resources (NCRR), and NIH Roadmap for Medical Research [U01 AR45580, U01 AR45614, U01 AR45632, U01 AR45647, U01 AR45654, U01 AR45583, U01 AG18197, U01-AG027810, and UL1 TR000128, K24-AR04884-06]. The National Heart, Lung, and Blood Institute (NHLBI) provides funding for the MrOS Sleep ancillary study "Outcomes of Sleep Disorders in Older Men" [R01 HL071194, R01 HL070848, R01 HL070847, R01 HL070842, R01 HL070841, R01 HL070837, R01 HL070838, and R01 HL070839]. The Sleep Heart Health Study (SHHS) is supported by the National Heart, Lung, and Blood Institute through the following cooperative agreements: U01-HL53940 (University of Washington), U01-HL53941 (Boston University), U01-HL63463 (Case Western Reserve University), U01-HL53937 (Johns Hopkins University), U01-HL53938 (University of Arizona), U01-HL53916 (University of California, Davis), U01-HL53934 (University of Minnesota), U01-HL63429 (Missouri Breaks Research), and U01-HL53931 (New York University). This work was also supported by philanthropic funding from Fan Hongbing (President of OMPA Corporation, Kaifeng, China) and the National Institutes of Health (R01HL102321, R01HL128658, P01HL095491, UL1RR025758). S.A.S. and L.T.-M. were supported by American Heart Association grants (15SDG25890059 and 17POST33410436, respectively); and partially supported by NHLBI R35HL135818 (to S.R., S.A.S., A.W., and A.A.).

Conflict of interest: A.A. serves as consultant for Somnifix. S.A.S. serves as consultant for Cambridge Sound Management, Nox Medical, and Merck. L.T.-M. served as a consultant for Novion Pharmaceuticals and Cambridge Sound Management. D.P.W. receives salary from Philips Respironics and is a consultant to Night Balance. A.W. works as a consultant for Somnifix, Cambridge Sound Management, Nox Medical, Bayer, Philips, and Galvani and has received grants from Philips and Somnifix. L.T.-M. and A.W. also have a financial interest in Apnimed Corp., a company developing pharmacologic therapies for sleep apnoea. Their interests were reviewed and are managed by Brigham and Women's Hospital and Partners HealthCare in accordance with their conflict of interest policies. S.A.I. is a consultant for Eisai, Merck, Acadia, Pfizer, GSK. K.L.S. has grant funding from Merck. L.M., K.E., and S.P. declare no conflicts of interest.

\section{References}

1. Young T, Peppard P, Gottlieb D. The epidemiology of obstructive sleep apnea: a population health perspective. Am J Respir Crit Care Med 2002;165:1217-1239.

2. Colt HG, Haas H, Rich GB. Hypoxemia vs sleep fragmentation as cause of excessive daytime sleepiness in obstructive sleep apnea. Chest 1991;100: 1542-1548.

3. Davies CWH, Crosby JH, Mullins RL, Barbour C, Davies RJ, Sradling JR. Casecontrol study of 24 hour ambulatory blood pressure in patients with obstructive sleep apnoea and normal matched control subjects. Thorax 2000;55:736-740.

4. White DP, Younes MK. Obstructive sleep apnea. Compr Physiol 2012;2: 2541-2594.

5. Engleman HM, Kingshott RN, Martin SE, Douglas NJ. Cognitive function in the sleep apnea/hypopnea syndrome (SAHS). Sleep 2000;23(Suppl 4):S102-S108.

6. Reynolds AC, Banks S. Total sleep deprivation, chronic sleep restriction and sleep disruption. Prog Brain Res 2010;185:91-103.

7. Nieto FJ, Young TB, Lind BK, Shahar E, Samet JM, Redline S, D'Agostino RB, Newman AB, Lebowitz MD, Pickering TG. Association of sleep-disordered breathing, sleep apnea, and hypertension in a large community-based study. Sleep Heart Health Study. JAMA 2000;283:1829-1836.

8. Peppard PE, Young T, Palta M, Skatrud J. Prospective study of the association between sleep-disordered breathing and hypertension. N Engl J Med 2000;342: 1378-1384.

9. Gottlieb DJ, Yenokyan G, Newman AB, O'Connor GT, Punjabi NM, Quan SF, Redline S, Resnick HE, Tong EK, Diener-West M, Shahar E. Prospective study of obstructive sleep apnea and incident coronary heart disease and heart failure: the sleep heart health study. Circulation 2010;122:352-360.

10. Marin JM, Carrizo SJ, Vicente E, Agusti AG. Long-term cardiovascular outcomes in men with obstructive sleep apnoea-hypopnoea with or without treatment with continuous positive airway pressure: an observational study. Lancet 2005; 365:1046-1053.

11. Punjabi NM, Beamer BA. Alterations in glucose disposal in sleep-disordered breathing. Am J Respir Crit Care Med 2009;179:235-240.

12. Kokkarinen J. Obstructive sleep apnea-hypopnea and incident stroke: the sleep heart health study. Am J Respir Crit Care Med 2011;183:950; author reply 950.

13. Redline S, Yenokyan G, Gottlieb DJ, Shahar E, O'Connor GT, Resnick HE, Diener-West M, Sanders MH, Wolf PA, Geraghty EM, Ali T, Lebowitz M, Punjabi NM. Obstructive sleep apnea-hypopnea and incident stroke: the sleep heart health study. Am J Respir Crit Care Med 2010;182:269-277.

14. Yaggi HK, Concato J, Kernan WN, Lichtman JH, Brass LM, Mohsenin V. Obstructive sleep apnea as a risk factor for stroke and death. N Engl J Med 2005; 353:2034-2041.

15. McEvoy RD, Antic NA, Heeley E, Luo Y, Ou Q, Zhang X, Mediano O, Chen R, Drager LF, Liu Z, Chen G, Du B, McArdle N, Mukherjee S, Tripathi M, Billot L, Li Q, Lorenzi-Filho G, Barbe F, Redline S, Wang J, Arima H, Neal B, White DP, Grunstein RR, Zhong N, Anderson CS. CPAP for prevention of cardiovascular events in obstructive sleep apnea. N Engl J Med 2016;375:919-931.

16. Kendzerska T, Gershon AS, Hawker G, Leung RS, Tomlinson G. Obstructive sleep apnea and risk of cardiovascular events and all-cause mortality: a decadelong historical cohort study. PLoS Med 2014;11:e1001599.

17. Jennum P, Tonnesen P, Ibsen R, Kjellberg J. All-cause mortality from obstructive sleep apnea in male and female patients with and without continuous positive airway pressure treatment: a registry study with 10 years of follow-up. Nat Sci Sleep 2015; 7:43-50.

18. Yu J, Zhou Z, McEvoy RD, Anderson CS, Rodgers A, Perkovic V, Neal B. Association of positive airway pressure with cardiovascular events and death in adults with sleep apnea: a systematic review and meta-analysis. JAMA 2017;318: 156-166.

19. Punjabi NM, Caffo BS, Goodwin JL, Gottlieb DJ, Newman AB, O'Connor GT, Rapoport DM, Redline S, Resnick HE, Robbins JA, Shahar E, Unruh ML, Samet 
JM. Sleep-disordered breathing and mortality: a prospective cohort study. PLoS Med 2009;6:e1000132.

20. Peker Y, Hedner J, Norum J, Kraiczi H, Carlson J. Increased incidence of cardiovascular disease in middle-aged men with obstructive sleep apnea: a 7-year follow-up. Am J Respir Crit Care Med 2002;166:159-165.

21. Oldenburg $O$, Wellmann B, Buchholz A, Bitter T, Fox H, Thiem U, Horstkotte $D$, Wegscheider K. Nocturnal hypoxaemia is associated with increased mortality in stable heart failure patients. Eur Heart J 2016;37:1695-1703.

22. Smagula SF, Stone KL, Redline S, Ancoli-Israel S, Barrett-Connor E, Lane NE, Orwoll ES, Cauley JA. Actigraphy- and polysomnography-measured sleep disturbances, inflammation, and mortality among older men. Psychosom Med 2016;78: 686-696.

23. Jung HH, Lee JH, Baek HJ, Kim SJ, Lee JJ. Nocturnal hypoxemia and periodic limb movement predict mortality in patients on maintenance hemodialysis. Clin J Am Soc Nephrol 2010;5:1607-1613.

24. Orwoll E, Blank JB, Barrett-Connor E, Cauley J, Cummings S, Ensrud K, Lewis C, Cawthon PM, Marcus R, Marshall LM, McGowan J, Phipps K, Sherman S, Stefanick ML, Stone K. Design and baseline characteristics of the osteoporotic fractures in men (MrOS) study - a large observational study of the determinants of fracture in older men. Contemp Clin Trials 2005;26:569-585.

25. Blank JB, Cawthon PM, Carrion-Petersen ML, Harper L, Johnson JP, Mitson E, Delay RR. Overview of recruitment for the osteoporotic fractures in men study (MrOS). Contemp Clin Trials 2005;26:557-568.

26. Dean DA, Goldberger AL, Mueller R, Kim M, Rueschman M, Mobley D, Sahoo SS, Jayapandian CP, Cui L, Morrical MG, Surovec S, Zhang G-Q, Redline S. Scaling up scientific discovery in sleep medicine: the National Sleep Research Resource. Sleep 2016;39:1151-1164.

27. Blackwell T, Yaffe K, Ancoli-lsrael S, Redline S, Ensrud KE, Stefanick ML, Laffan A, Stone KL. Associations between sleep architecture and sleep-disordered breathing and cognition in older community-dwelling men: the Osteoporotic Fractures in Men Sleep Study. J Am Geriatr Soc 2011;59:2217-2225.

28. Quan SF, Howard BV, Iber C, Kiley JP, Nieto FJ, O'Connor GT, Rapoport DM, Redline S, Robbins J, Samet JM, Wahl PW. The Sleep Heart Health Study: design, rationale, and methods. Sleep 1997;20:1077-1085.

29. Redline S, Sanders MH, Lind BK, Quan SF, Iber C, Gottlieb DJ, Bonekat WH, Rapoport DM, Smith PL, Kiley JP. Methods for obtaining and analyzing unattended polysomnography data for a multicenter study. Sleep Heart Health Research Group. Sleep 1998;21:759-767.

30. Mehra R, Stone KL, Blackwell T, Ancoli Israel S, Dam T-TL, Stefanick ML, Redline $\mathrm{S}$. Prevalence and correlates of sleep-disordered breathing in older men: osteoporotic fractures in men sleep study. J Am Geriatr Soc 2007;55:1356-1364.

31. Berry RB, Budhiraja R, Gottlieb DJ, Gozal D, lber C, Kapur VK. Rules for scoring respiratory events in sleep: update of the 2007 AASM Manual for the Scoring of Sleep and Associated Events. Deliberations of the Sleep Apnea Definitions Task Force of the American Academy of Sleep Medicine. J Clin Sleep Med 2012;8:597-619.

32. Sheather SJ. A Modern Approach to Regression with R. New York: Springer; 2009.
33. Therneau TM, Crowson CS, Atkinson EJ. Adjusted Survival Curves. 2015. https:// cranr-projectorg/web/packages/survival/vignettes/adjcurvepdf (4 October 2018).

34. Putcha N, Crainiceanu C, Norato G, Samet J, Quan SF, Gottlieb DJ, Redline S, Punjabi NM. Influence of lung function and sleep-disordered breathing on all-cause mortality. A community-based study. Am J Respir Crit Care Med 2016;194:1007-1014.

35. Dewan NA, Nieto FJ, Somers VK. Intermittent hypoxemia and OSA: implications for comorbidities. Chest 2015;147:266-274.

36. Sert Kuniyoshi FH, Singh P, Gami AS, Garcia-Touchard A, van der Walt C, Pusalavidyasagar S, Wright RS, Vasquez EC, Lopez-Jimenez F, Somers VK. Patients with obstructive sleep apnea exhibit impaired endothelial function after myocardial infarction. Chest 2011;140:62-67.

37. Somers VK, Mark AL, Zavala DC, Abboud FM. Contrasting effects of hypoxia and hypercapnia on ventilation and sympathetic activity in humans. J Appl Physiol (1985) 1989;67:2101-2106.

38. Jelic S, Lederer DJ, Adams T, Padeletti M, Colombo PC, Factor PH, Le Jemtel TH. Vascular inflammation in obesity and sleep apnea. Circulation 2010;121: 1014-1021.

39. Lavie L, Lavie P. Molecular mechanisms of cardiovascular disease in OSAHS: the oxidative stress link. Eur Respir J 2009;33:1467-1484.

40. Levy P, Bonsignore MR, Eckel J. Sleep, sleep-disordered breathing and metabolic consequences. Eur Respir J 2009;34:243-260.

41. Gami AS, Hodge DO, Herges RM, Olson EJ, Nykodym J, Kara T, Somers VK. Obstructive sleep apnea, obesity, and the risk of incident atrial fibrillation. J Am Coll Cardiol 2007;49:565-571.

42. Gami AS, Olson EJ, Shen WK, Wright RS, Ballman KV, Hodge DO, Herges RM, Howard DE, Somers VK. Obstructive sleep apnea and the risk of sudden cardiac death: a longitudinal study of 10,701 adults. J Am Coll Cardiol 2013;62:610-616.

43. Punjabi NM. COUNTERPOINT: is the apnea-hypopnea index the best way to quantify the severity of sleep-disordered breathing? No. Chest 2016;149:16-19.

44. Cowie MR, Woehrle H, Wegscheider K, Angermann C, d'Ortho M-P, Erdmann E, Levy P, Simonds AK, Somers VK, Zannad F, Teschler H. Adaptive servoventilation for central sleep apnea in systolic heart failure. N Engl J Med 2015; 373:1095-1105

45. Mokhlesi B, Ayas NT. Cardiovascular events in obstructive sleep apnea-can CPAP therapy SAVE lives? N Engl J Med 2016;375:994-996.

46. Azarbarzin A, Ostrowski M, Hanly P, Younes M. Relationship between arousal intensity and heart rate response to arousal. Sleep 2014;37:645-653.

47. Azarbarzin A, Ostrowski M, Younes M, Keenan BT, Pack AI, Staley B, Kuna ST. Arousal responses during overnight polysomnography and their reproducibility in healthy young adults. Sleep 2015;38:1313-1321.

48. Amatoury J, Azarbarzin A, Younes M, Jordan AS, Wellman A, Eckert DJ. Arousal intensity is a distinct pathophysiological trait in obstructive sleep apnea. Sleep 2016;39:2091-2100.

49. Younes M, Ostrowski M, Soiferman M, Younes H, Younes M, Raneri J, Hanly P. Odds ratio product of sleep EEG as a continuous measure of sleep state. Sleep 2015;38:641-654.

\section{Corrigendum}

Corrigendum to: The hypoxic burden of sleep apnoea predicts cardiovascular disease-related mortality: the Osteoporotic Fractures in Men Study and the Sleep Heart Health Study [Eur Heart] 2019;40:1149-1157].

During a subsequent analysis of one of the cohorts analyzed and reported in the above paper, the authors discovered that some of the respiratory events were mislabeled by the polysomnography system in one of the two cohorts (MrOS) and were not included in the analysis of hypoxic burden. The analysis for the MrOS cohort was repeated using the corrected hypoxic burden index (Sleep Heart Health Study data were unaffected by this). The point estimates for the paper's main models got somewhat stronger in the re-analyzed data, but did not materially change the interpretation of the paper. The paper has now been amended accordingly in print and online. The supplementary material online has also been amended.

The authors apologise for the error. 